International Journal of Pure and Applied Mathematics

Volume 107 No. 4 2016, 1025-1036

ISSN: 1311-8080 (printed version); ISSN: 1314-3395 (on-line version)

url: http://www.ijpam.eu

doi: 10.12732/ijpam.v107i4.20

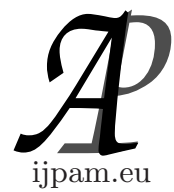

\title{
PERFORMANCE OF SELF-ORGANIZED AND METACOGNITIVE NEUROFUZZY SYSTEM FOR TRAFFIC FLOW PREDICTION
}

\author{
Kesavan Asaithambi ${ }^{1}$, A. Nagoor Gani ${ }^{2}$ \\ 1,2 Jamal Mohamed College (Autonomous) \\ Tiruchy, INDIA
}

\begin{abstract}
Accurate prediction of traffic flow is an important step needed in urban traffic management systems. While several neurofuzzy approaches have been used in literature for this particular problem, most of them need manual intervention in the formulation of the fuzzy rule base and also in determining the architecture of the neurofuzzy system. This paper evaluates two recent neurofuzzy algorithms that are capable of automatically determining the rule base and architecture in a purely data driven approach. An open source traffic data has been evaluate and compare the performance of these neurofuzzy systems.
\end{abstract}

Key Words: system identification, neuro-fuzzy inference system, traffic flow prediction, meta-cognition

\section{Introduction}

In transportation and logistics travel time information plays an important role. Nowadays it is applied in applied in various Intelligent Transport Systems (ITS), such as in-vehicle route guidance (RGS) and advanced traffic management systems (ATMS). A range of different traffic factors affect the travel time. A large traffic data is required to accurately predict the travel time. A good Understanding of the traffic factors affecting travel time is necessary for improving prediction accuracy in related travel time studies.

Received: February 27, 2016

Published: May 7, 2016

${ }^{\S}$ Correspondence author (c) 2016 Academic Publications, Ltd.

url: www.acadpubl.eu 
A major problem in big cities in developed and developing countries is traffic congestion. Different approaches (more or less successful) have been used to reduce congestions. Research reveals that traffic congestions can be reduced by providing accurate traffic information to drivers promptly. This information helps drivers to choose alternative route, avoid congested roads or even change departure time. The intelligent transport systems (ITS) that predict congestions help traffic control to eliminate congestions, or relay the information about future congestion so that motorists may change their decisions accordingly. Different sensor systems like embedded roadway loop detectors, electronic toll collection transponders, automatic vehicle location, etc are used by Modern ITS to collect data about actual traffic. These ITS systems can predict congestion and even estimate route duration based on this sensory data.

Kamarianakis and Prastacos [4] estimate parameters in a model which takes into account spatial and temporal correlations across the road network. Even though the basic form of their model has some similarity to ours, noticeable differences exist. Added to this the authors in [4] assume stationarity of the system. Though the traffic flow parameters are clearly not stationary over the time period being modelled, the authors propose to perform differencing of the data points, with a differencing period of one day. And inter-day fluctuations are not considered, which should violate the stationarity assumption and introduce non-negligible bias into the estimated parameters.

Real-time traffic flow prediction has been studied in several earlier works using neural networks, such as $[1,2,3]$. [3] examines 15-minute traffic volumes during daytime in an expressway, and predicts the volume for the next 15minute interval using a combination of methods, including neural networks. MNN - modular neural network is based on a divide-and-conquer strategy [5]. The input gets processed in several sub networks, each specializing in a specific task. MNNs are quicker to train and can improve results $[6,7,8,9]$. Hidden layer of so called basis functions to cluster the input space, with each cluster represented by a hidden neuron is used in RBFNN (Radial basis frequency networks). Results indicate a slightly positive preference of RBFNN over BPNN $[10,11,12,13]$. Fuzzy rules are defined automatically $[14,15,16]$ on the basis of data, in the hidden layer of neuro-fuzzy network. The results are better and even comparable to those of the BPNN. In a counter propagation network, every time the inputs during iteration are assigned to one node using a distance measure [17].Training time gets drastically decreased and performance is slightly better. Hidden units are created automatically in a resource allocating network and except that it is almost like the RBFNN [18].

Recently, a Self-Adaptive Fuzzy Inference Network (SaFIN) have been de- 
veloped in [20]. SaFIN addresses the problem of self-organization in a fuzzy neural network using a novel cluster identification method and an automated rulebase generation. SaFIN was applied to the traffic-flow prediction problem using data obtained from the road network in Singapore and it was found to perform better than the tradition fuzzy-neural approaches. However, SaFIN does not use the concept of meta-cognition. All te samples in the training set are used in the learning process of SaFIN. Recent developments in machine learning suggest that, meta-cogitive learning concepts addressing the issues of what to learn, when to learn and how to learn provide enhanced learning performance. Recently, a Metacognitive interval type-2 neurofuzzy inference system (McIT2FIS) was developed in [25] and it was applied to several regression and system identification problems successfuly. In this paper we used road network data of the United Kingdom (UK) [19], to study the traffic flow prediction problem using the recently developed McIT2FIS and the SaFIN neurofuzzy networks. The results show that both these systems are capable of accurately predicting the traffic flow, while the McIT2FIS provides higher accuracy that may be attributed to its meta-cognitive learning capability.

Paper organization: The next section (Section 2) provides an overview of the approach used in this paper for traffic flow prediction. This section also describes the dataset used and the working principles of the SaFIN and McIT2FIS neurofuzzy algorithms. Section 3 provides the details of the experimental results obtained by applying the above said neurofuzzy networks for the traffic prediction problem. The conclusions from the study are summarized in the Section 4.

\section{Materials and Methods}

\subsection{Dataset}

Data.gov.uk [19] is an intiative of the government of the UK which provides open sharing of transport data across the road network in UK using the Highways Agency network journey time and traffic flow data. This data is publishes by Highways Agency under the Open Government Licence. This data series provides average journey time, speed, link coordinates and traffic flow information for 15-minute periods since April 2009 on all motor ways and 'A' roads managed by the Highways Agency, known as the Strategic Road Network, in England. Journey times and speeds are estimated using a combination of sources, including Automatic Number Plate Recognition (ANPR) cameras, in- 
vehicle Global Positioning Systems (GPS) and inductive loops built into the road surface. While the link coordinates refer to as all-numeric easting and northing. Latitude and longitude coordinates based on the World Geodetic System (WGS84). The syntax of the available data is provided in table 1.

Table 1: Data definitions for the monthly Strategic Road Network $(\mathrm{SRN})$ data

\begin{tabular}{|c|c|}
\hline $\begin{array}{l}\text { Variable } \\
\text { name }\end{array}$ & Variable description \\
\hline LinkRef & $\begin{array}{l}\text { A unique alphanumeric link id representing a junction to junction } \\
\text { link on the Highways Agency managed road network. }\end{array}$ \\
\hline $\begin{array}{l}\text { Link De- } \\
\text { scription }\end{array}$ & Description of the link. \\
\hline Date & Date of travel. \\
\hline TimePeriod & $\begin{array}{l}\text { One of } 9615 \text {-minute intervals in the day that the data refers to } \\
(0-95 \text { where } 0 \text { indicates } 00: 00 \text { to } 00: 15) \text {. }\end{array}$ \\
\hline Average JT & $\begin{array}{l}\text { The average journey time to travel across the LinkRef in seconds, } \\
\text { of vehicles entering the junction to junction link within a given } \\
\text { 15-minute time period. }\end{array}$ \\
\hline $\begin{array}{l}\text { Average } \\
\text { Speed }\end{array}$ & $\begin{array}{l}\text { The average speed (in } \mathrm{km} / \mathrm{h} \text { ) of vehicles entering the junction to } \\
\text { junction link within a given } 15 \text {-minute time period. }\end{array}$ \\
\hline Data Quality & $\begin{array}{l}\text { Indicator showing the quality of the journey time data for the link } \\
\text { and time period. } 1 \text { indicates the highest quality data and } 5 \text { the } \\
\text { lowest. } \\
1=\text { Observed or vertically in-filled* data with a good spatial } \\
\text { match* to the link } \\
2=\text { Observed or vertically in-filled data with a poor spatial match } \\
\text { to the link. } \\
3=\text { Horizontally* in-filled data with a good spatial match to the } \\
\text { link. } \\
4=\text { Horizontally in-filled data with a poor spatial match to the } \\
\text { link. } \\
5=\text { No observed data so data are in-filled using free-flow data. } \\
\text { The length of the link (km). }\end{array}$ \\
\hline Flow & $\begin{array}{l}\text { An average of the observed flow for the link, time period and day } \\
\text { type. }\end{array}$ \\
\hline
\end{tabular}

* Vertical in-filling uses observed journey time data from adjacent time periods on the same day and link. Spatial match measures how precisely the source data maps onto the particular road link. For example, a pair of ANPR cameras that covered only a small portion of a complete junction-to-junction link may be reported as having a poor spatial match.Horizontal in-filling uses observed journey time data from equivalent time intervals on different dates of the same day type and link. 


\subsection{Traffic Flow Prediction as a System Identification Problem}

The problem of predicting the trafic flow an be viewed as a system identification problem. In this approach, the predicted value of the traffic flow $(\hat{y}(t+1))$ for the next time period is assumed to be a function of the traffic flow obtained during the past $F$ time periods viz $(y(t), y(t-1), y(t-2), \ldots, y(t-F+1))$.

\subsection{Type 1-Neurofuzzy System: SaFIN}

Traditional neural fuzzy systems the are designed by human experts are found to be highly subjective. Hence, self-organizing neural fuzzy systems that can automatically determine their architecture and parameters based on the numerical training data are being researched. Some common challenges for such self-organizing neural fuzzy systems are the consistency of the rulebase, apriori knowledge (Eg. clusters) and the considerations on the stabilityplasticity tradeoff. Self-Adaptive Fuzzy Inference Network (SaFIN) [20], is a novel self-organizing neural fuzzy system, that address the aforementioned deficiencies. SaFIN model employs categorical learning induced partitioning (CLIP), to automatically determine the number of clusters. CLIP is inspired by the behavioral category learning process in by human beings. Using CLIP, SaFIN is able to incorporate new clusters to provide a satisfactory representation of the incoming training data along every inputoutput dimension. This allows SaFIN the flexibility to incorporate new knowledge with old knowledge in the system. In addition, a consistent rulebase is also obtained in SaFIN using an automated rule formation mechanism. SaFIN model has been evaluated on a series of benchmark simulations to demonstrate its efficiency.

\subsubsection{Learning Algorithm of SaFIN}

SaFIN uses a five layer neurofuzzy architecture to perform the system identification task and predict the traffic flow. The function of each layer is provided in Table 2. The two main steps in the learning process of SaFIN are the automated fuzzy partitioning and the automatic rulebase generation. Initially, hidden layers 2 to 4 do not contain any nodes and they are slowly built up during the training process. Fuzzy labels are created on the input and output dimension based on the knowledge extracted from the data. The numbers, positions, and spreads of the fuzzy labels are self-determined from the training dataset. New clusters that may represent the new knowledge extracted from each training sample are created and also the existing clusters are refined, to 
address the trade-off between stability and plasticity simultaneously.

Table 2: Architecture of SaFIN [20] and McIT2FIS [25]

\begin{tabular}{|l|l|l|}
\hline & SaFIN & McIT2FIS \\
\hline Layer1 & $\begin{array}{l}\text { The input nodes do not perform any processing and they } \\
\text { directly pass the input to the next layer. }\end{array}$ \\
\hline Layer2 & $\begin{array}{l}\text { The fuzzy labels define the an- } \\
\text { tecedent sections of the Type } \\
1 \text { Mamdani-type rules. }\end{array}$ & $\begin{array}{l}\text { In this layer each ode fuzzi- } \\
\text { fies the input by employing an } \\
\text { interval-2 Gaussian member- } \\
\text { ship function. }\end{array}$ \\
\hline Layer3 & $\begin{array}{l}\text { A set of fuzzy rules (Mam- } \\
\text { dani/Type 1) extracted based } \\
\text { on training data is defined in } \\
\text { the rule layer of the SaFIN } \\
\text { model. }\end{array}$ & $\begin{array}{l}\text { In this layer each node repre- } \\
\text { sents the upper and lower fir- } \\
\text { ing strength of each of the K } \\
\text { rules. }\end{array}$ \\
\hline Layer4 & $\begin{array}{l}\text { The consequents correspond- } \\
\text { ing to the Type1 Mamdani } \\
\text { fuzzy rules in the system are } \\
\text { encoded in this layer. }\end{array}$ & $\begin{array}{l}\text { This layer each node reduces } \\
\text { the interval type-1 fuzzy set to } \\
\text { a type-1 fuzzy number. }\end{array}$ \\
\hline Layer5 & $\begin{array}{l}\text { The output nodes obtain a } \\
\text { crisp output value via defuzzi- } \\
\text { fication. }\end{array}$ & $\begin{array}{l}\text { This layer find out the de- } \\
\text { fuzzified output of the infer- } \\
\text { ence system by employing the } \\
\text { weighted average defuzzifica- } \\
\text { tion method. }\end{array}$ \\
\hline
\end{tabular}

Further details of the architecture and learning process in SaFIN can be found in $[20]$.

\subsection{Interval Type 2-Neurofuzzy System: McIT2FIS}

In the literature, interval type-2 neurofuzzy inference systems (IT2FIS), have been developed recently [23, 24]. The Metacognitive interval type-2 neurofuzzy inference system (McIT2FIS) [25] is also a recently developed neuro fuzzy system that is based on interval type 2 fuzzy inference which achieves fast intervalreduction using a metacognitive sequential learning algorithm. During learning, McIT2FIS automatically creates the fuzzy rules that are necessary for effective learning during the training process from the data itself.Initially, there are no fuzzy rules in McIT2FIS and as a new sample is presented it evolves the structure and parameters by itself based on novel information embedded 
within the sample. Whenever a new rule is added to the network to represent such new knowledge or information, it is done in a manner that exploits the localization property of Gaussian rules [22]. The various parameters of the rules are adapted by employing an extended Kalman filtering (EKF) based approach. McIT2FIS employs self-regulated learning by deciding what-to-learn, when-tolearn, and how-to-learn to effectively capture the underlying data distribution. This strategy also prevents overtraining. The metacognitive component of McIT2FIS controls the learning process by deciding on an efficient learning strategy.

\subsubsection{Learning Algorithm of McIT2FIS}

McIT2FIS also uses a five layer neuro fuzzy architecture and the function of each layer is provided in Table 2.

Knowledge measures: The McIT2FIS uses two measures to indicate the new knowledge embedded within a training sample. First is the prediction error given by $\left(\sqrt{(\hat{y}(t)-y(t))^{2}}\right)$ and the spherical potential as defined in [22].

Based on the knowledge measures, McIT2FIS chooses one of the following strategies for each new training smaple presented to it during the learning process.

Sample deletion: If the sample has little or no new knowledge as shown by the measures above (using some thresholds), then the sample is deleted from the training sequence and it is not used for training.

Sample Learning: If there is a significant new knowledge in the training sample beyond a certain threshold, then a new rule may be created. If there is only limited new knowledge, then only the parameters of the network are updated.

Sample reserve: If the criteria for both the above strategies are not satisfied, then the sample is reserved for future use.

Further details of the architecture and learning process in McIT2FIS can be found in $[25]$.

\section{Results}

In this section, the performance of the SaFIN and McIT2FIS algorithms are evaluated for the traffic flow prediction problem using the dataset provided in [19]. From this comprehensive dataset, the traffic flow information for the month of March 2015 was extracted for the link number AL100, which represent 


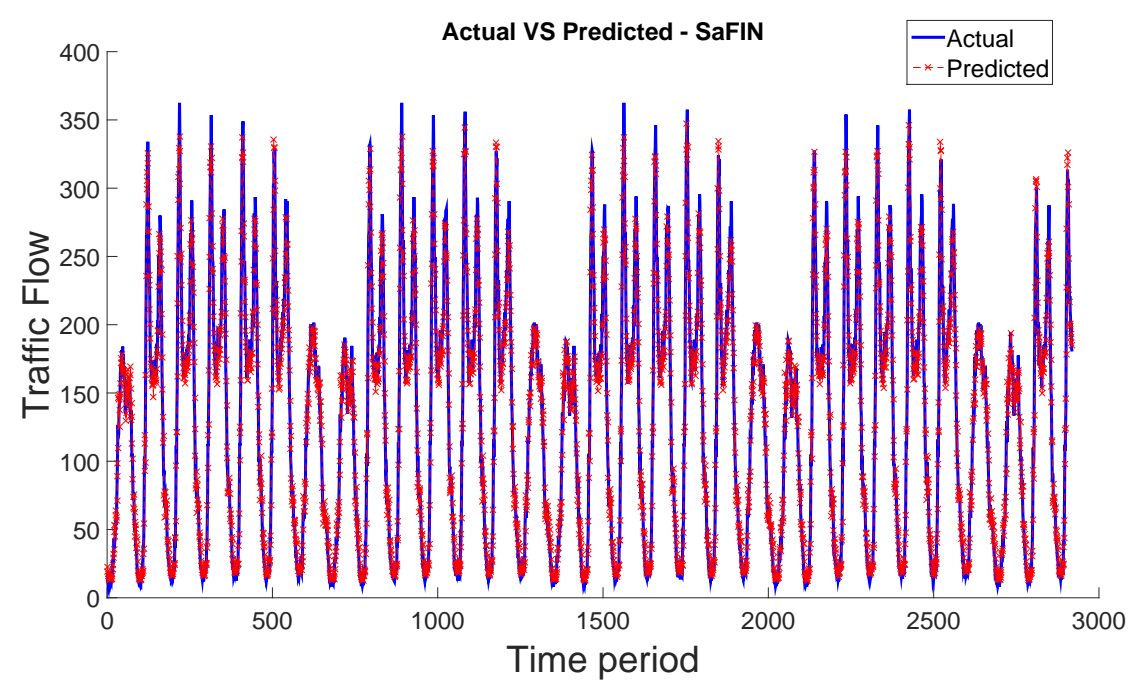

Figure 1: Performance of SaFIN for traffic flow prediction

the section of the road A414 between M1 J7 and A405. The dataset provides the traffic flow in this link for about 95 time periods in the day for the entire month of March 2015. Using the system identification approach mentioned earlier, the traffic flow data for the past 6 time periods are now used to predict the traffic flow for the next time period using SaFIN and McIT2FIS approaches. all the simulations were performed using MATLAB 2014 running on a computer with an intel i7 processor with 16 GB of RAM.

\subsection{Performance of SaFIN}

The actual flow and the predicted values of traffic flow, obtained from SaFIN are plotted in fig 1. It can be observed that the predicted value traces the actual values very closely. Thus, SaFIN is able to effectively identify the system and provide accurate prediction of the traffic flow. To evaluate the accuracy, the Pearson correlation co-efficient $(\mathrm{R})$ was calculated between the actual and the predicted outputs. A value of 0.98 was obtained for (R), indicating a strong correlation between the actual and predicted values. The mean square error (MSE) between the predicted and actual outputs were also computed and a value of 169.22 was obtained. It is possible to improve this performance by normalization of the data.

For the system identification task, the SaFIN used three terms for fuzzy 
clustering of each input and output dimension as shown in fig 2. A total of about 54 fuzzy rules were created automatically by SaFIN to perform the system identification task.

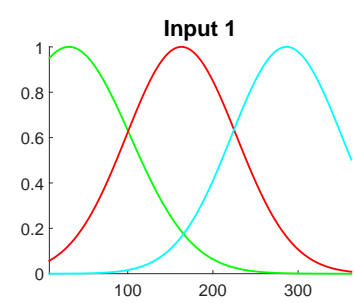

(a) Input 1

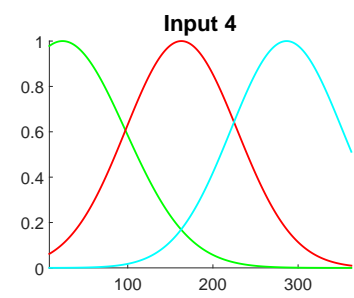

(d) Input 4

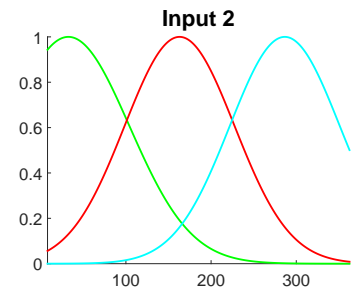

(b) Input 2

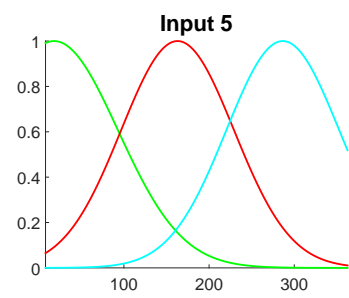

(e) Input 5

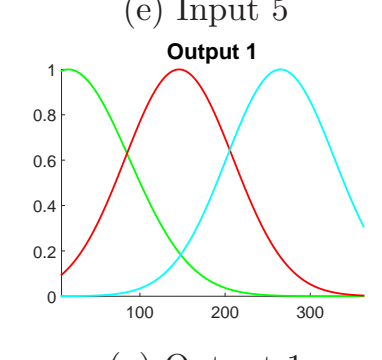

(g) Output 1

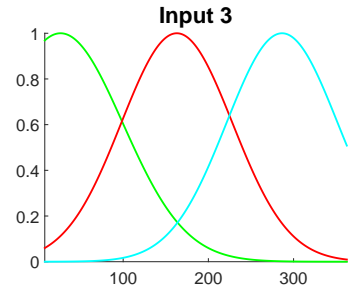

(c) Input 3

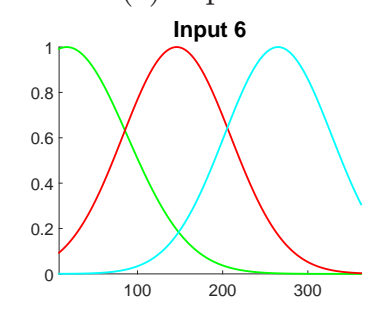

(f) Input 6

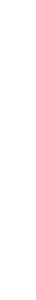




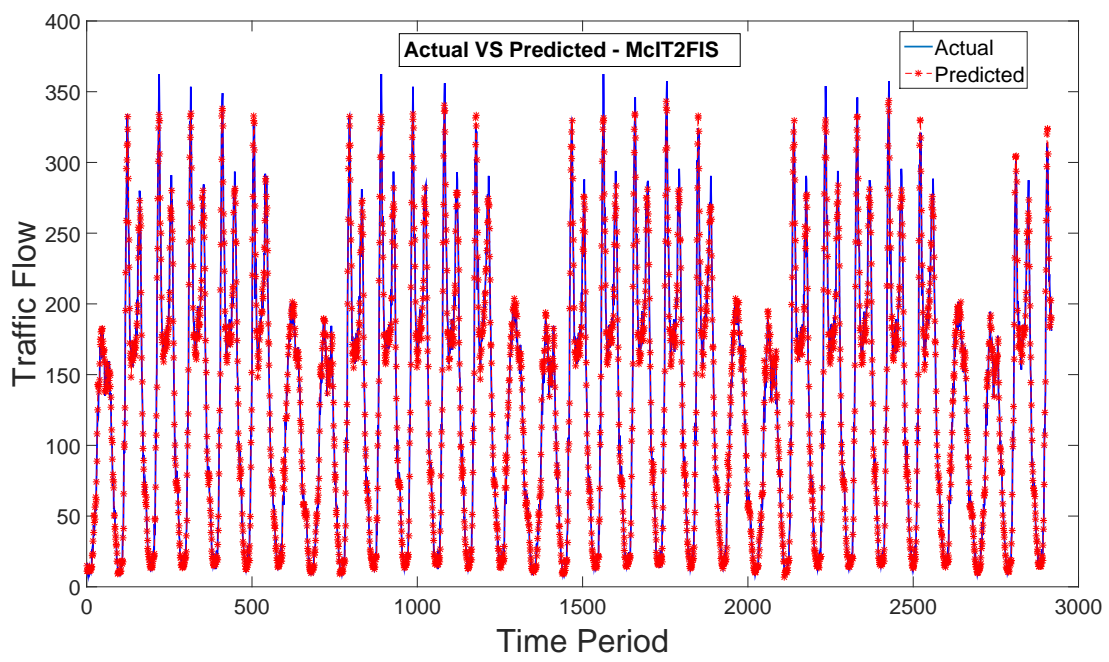

Figure 3: Performance of McIT2FIS for traffic flow prediction

by McIT2FIS was 92.76. This is also lower than the error obtained for SaFIN. Thus it is clear that McIT2FIS is able to provide more accurate prediction of the traffic flow with the help of meta-cognitive learning strategies. A total of 57 rules were created automatically by McIT2FIS to perform the system identification task.

\section{Conclusions}

This paper studied the application of two recent neuro fuzzy approaches to the traffic flow prediction problem using an open source traffic data. Among these approaches, the SaFIN is a type 1 fuzzy inferences system with self organizing capabilities while the McIT2FIS is an interval type 2 fuzzy inference system, with meta-cognitive learning capabilities. both these network are capable of automatically determining their architecture and rulebases in a purely data driven approach. This eliminated the need for a manual intervention in the creation of fuzzy rules and the design on the network. Among the two approaches, the McIT2FIS is able to achieve lower error due to its meta-cognitive learning capabilities. 


\section{References}

[1] Vlahogianni, E.I.; Karlaftis, M.G.; Golias, J.C.; Optimized and meta-optimized neural networks for short-term traffic flow prediction: A genetic approach, Transportation Research Part C:Emerging Technologies, 13 (3),(2005) 211-234.

[2] Wang, Y.; Papageorgiou, M.; and Messmer, A.; Real-Time Freeway Traffic State Estimation Based on Extended Kalman Filter: A Case Study. Transportation Science, 41(2), (2007), 167-181.

[3] Zheng, W.; Lee, D.-H.; and Shi, Q; Short-Term Freeway Traffic Flow Prediction: Bayesian Combined Neural Network Approach, Journal of Transportation Engineering, 132(2), (2006), 114-121.

[4] Kamarianakis, Y; and Prastacos, P.; Forecasting traffic flow conditions in an urban network: comparison of multivariate and univariate approaches. In Transportation Research Record, Journal of the Transportation Research Board, 1857, (2003), 74-84.

[5] Ishak, S., and C. Alecsandru. Optimizing Traffic Prediction Performance of Neural Networks Under Various Topological, Input, and Traffic Condition Settings. Journal of Transportation Engineering, 130(4), 2004, 452-465.

[6] Alecsandru, C.-D. A Hyrbid Model-Based and Memory-Based Short-Term Traffic Prediction System. Louisiana State University and Agricultural and Mechanical College, 2003.

[7] Ledoux, C. An Urban Traffic Flow Model Integrating Neural Networks. Transportation Research Part C, Vol. 5, No. 5, 1997, pp. 287300.

[8] Zheng, W., D.-H. Lee, and Q. Shi. Short-Term Freeway Traffic Flow Prediction: Bayesian Combined Neural Network Approach. Journal of Transportation Engineering, Vol. 132, No. 2, 2006, pp. 114-121.

[9] Zhang, H., S. G. Ritchie, and Z.-P. Lo. Macroscopic Modeling of Freeway Traffic Using an Artificial Neural Network. In Transportation Research Record 1588, TRB, National Research Council, Washington, D.C., 2000, pp. 110-119.

[10] Park, B., C. J. Messer, and T. Urbanik II. Short-Term Freeway Traffic Volume Forecasting Using Radial Basis Function Neural Networks. In: Transportation Research Record 1651, TRB, National Research Council, Washington, D.C., 1998, pp. 39-47.

[11] Amin, S. M., E. Y. Rodin, A.-P. Liu, K. Rink, and A. Carcia-Ortiz. Traffic Prediction and Management Via RBF Neural Nets and Semantic Control. Computer-Aided Civil and Infrastructure Engineering, Vol. 13, 1998, 315-327. 131.

[12] Huisken, G. Soft-Computing Techniques Applied to Short-Term Traffic Flow Forecasting. Systems Analysis Modelling Simulation, Vol. 43, No. 2, 2003, pp. 165-173. 132.

[13] Xie, Y., and Y. Zhang. A Wavelet Network Model for Short-Term Traffic Volume Forecasting. Journal of Intelligent Transportation Systems, Vol. 10, No. 3, 2006, pp. 141-150.

[14] Coufal, D., and E. Turunen. Short Term Prediction of Highway Travel Time Using Data Mining and Neuro-Fuzzy Methods. Neural Network World, Vol. 34, 2004, pp. 221-231. 134.

[15] Xiao, H., H. Sun, and B. Ran. The Special Factor Adjustment Model Using Fuzzy-Neural Network in Traffic Prediction. Presented at 83rd Annual Meeting of the Transportation Research Board, Washington, D.C., 2004. 135. 
[16] Yin, H., S. C. Wong, J. Xu, and C. K. Wong. Urban Traffic Flow Prediction Using a Fuzzy-Neural Approach. Transportation Research Part C, Vol. 10, 2002, pp. 85-98.

[17] Dharia, A., and H. Adeli. Neural Network Model for Rapid Forecasting of Freeway Link Travel Time. Engineering Applications of Artificial Intelligence, Vol. 16, 2003, pp. 607613.

[18] Chen, H., and S. Grant-Muller. Use of Sequential Learning for Short-Term Traffic Flow Forecasting. Transportation Research Part C, Vol. 9, 2001, pp. 319-336.

[19] https://data.gov.uk/dataset/gb-road-traffic-counts/

[20] Tung, Sau Wai and Quek, Chai and Guan, Cuntai; SaFIN: A self-adaptive fuzzy inference network, IEEE Transactions on Neural Networks, vol. 22 No. 12 , 1928-1940, 2011.

[21] K. Subramanian and S. Suresh, A meta-cognitive sequential learning algorithm for neurofuzzy inference system, Applied Soft Computing, vol. 12, no. 11, pp. 3603-3614, 2012.

[22] K. Subramanian, S. Suresh, and N. Sundararajan, A meta-cognitive neuro-fuzzy inference system (MCFIS) for sequential classification problems, IEEE Transactions on Fuzzy Systems, vol. 21, no. 6, pp. 1080-1095, Dec. 2013.

[23] C. Lee, J. Hong, Y. Lin, andW. Lai, Type-2 fuzzy neural network systems and learning, International Journal of Computational Cognition, vol. 1, no. 4, pp. 79-90, 2003.

[24] C.-F. Juang and P.Wang, An interval type-2 neural fuzzy classifier learned through soft margin minimization and its human posture classification application, IEEE Transactions on Fuzzy Systems, vol. 23 No. 5 , 1474-1487, 2015.

[25] Ankit Kumar Das, Kartick Subramanian, and Suresh Sundaram, An Evolving Interval Type-2 Neurofuzzy Inference System and Its Metacognitive Sequential Learning Algorithm, IEEE Transactions on Fuzzy Systems, vol. 23 No. 6 , 2080-2093, 2015. 